\title{
Multi-constellation GBAS: how to benefit from a second constellation
}

\author{
Maria Caamano, Michael Felux, Mihaela-Simona Circiu and Daniel Gerbeth \\ German Aerospace Center (DLR) \\ Institute of Communications and Navigation \\ 82234 Wessling-Oberpfaffenhofen, Germany \\ \{Maria.caamanoalbuerne, Michael.felux, Mihaela-simona.circiu, Daniel.gerbeth\}@dlr.de
}

\begin{abstract}
In this paper we analyze and discuss the impact of ionospheric scintillations and multipath on the availability of the current single-frequency single-constellation Ground Based Augmentation System (GBAS). Scintillation effects, which usually occur around plasma bubbles, cause the receiver to lose lock of one or more satellites, leading to potentially unfavorable satellite geometries for an airborne user. We simulate different bubble dimensions and distinct locations of the bubble in the sky in order to illustrate that the use of a second constellation improves the performance of the system in terms of availability for the situations where the single constellation system is unavailable. Furthermore, we investigate the impact of multipath in the availability of the system. During the touch-down and rollout of the aircraft on the runway, multipath coming from ground reflections becomes important, especially for low elevation satellites producing large position errors. The results show that the use of a second constellation allows the removal of low elevation satellites from the position solution, which are typically strongly affected by multipath. Elevation masks of $10^{\circ}$ or even $15^{\circ}$ do not degrade the availability of the dual-constellation system in any of the scenarios considered.
\end{abstract}

Index Terms-plasma bubbles, multipath, availability.

\section{INTRODUCTION}

The current fully operational Global Navigation Satellite Systems (GNSS), GPS and GLONASS, were not designed to meet the real-time integrity requirements for civil aviation navigation safety needs. This implies that if a pseudorange measurement is affected by error such ionospheric disturbances or significant multipath, these satellite systems have no way to detect a potentially hazardous condition and warn a user. For this purpose augmentation systems were developed to increase the accuracy of GNSS navigation and provide integrity such that the required safety standards in aviation can be met.

The Ground Based Augmentation System (GBAS) is a development of local-area differential GNSS whose main purpose is to provide precision approach guidance for aircraft. It consists of a ground subsystem and an airborne subsystem. The ground subsystem provides the aircraft with approach path data and, for each satellite in view, corrections and integrity information. The corrections enable the aircraft to determine its position relative to the approach path very accurately while the integrity parameters enable the bounding of the residual position errors and ensure safety of the operation.
Current commercially available GBAS stations are based on a single-frequency (L1) single-constellation (GPS) architecture. However, the use of a single constellation may lead, under certain conditions, to a degraded availability of the navigation service. Generally, these conditions could be caused by one of the following problems: (i) the blocking of the signals coming from the satellites, (ii) the conservative assumptions due to the bounding of the worst-experienced error and (iii) the exclusion of the satellites that could be affected by errors that up to date are not mitigatable. We describe in the next paragraphs each of these issues.

The loss of GNSS signals coming from low-elevation satellites may be caused by banking-maneuvers. The antenna located on top of the aircraft might not receive the signals blocked by some parts of the plane; e.g. the wings. This situation could cause an unfavorable geometry due to a smaller number of satellites available to calculate the position solution of the aircraft [1]. A different example may occur due to required masking for non-optimal GBAS reference sites, e.g. the landscape of airports with many buildings near the ground station, which potentially also reduces the number of satellites available for navigation.

The ionospheric delay gradients acting between the ground station and the airplane on approach may result in nondifferentially corrected errors in GBAS. Protection of airborne users against this kind of threat requires either very conservative error bounding by inflation of the integrity parameters (the strategy in GBAS Approach Service Type (GAST) C) or a significant amount of sensitive monitoring (the strategy in GAST D). These two methods are able to ensure the safety of the users, but could result in undesirable unavailability of the system [2].

Ionospheric scintillations are rapid variations of the GNSS signals due to small scale ionospheric irregularities, e.g. ionospheric plasma bubbles. The GBAS reference receivers as well as airborne users are often heavily affected by scintillations as the receivers cannot maintain a continuous tracking of the affected signals [2]. Another concern is the multipath coming from ground reflections, which arises especially during touchdown and roll-out of the aircraft on the runway. It affects 
the airborne measurements, especially during those phases, driving the required performance to its limits in current GBAS setups [3].

The first two examples are strongly connected to the structure of the specific aircraft, or to the specific conditions and infrastructure of the airport, which makes a detailed analysis intractable. The ionospheric gradient issue is already treated as we have mentioned. That is the main reason why in this work, we focus on the impact that both ionospheric scintillations and multipath have on the availability of current single-frequency single-constellation GBAS. Furthermore, we compare it with the performance when using additionally a second constellation, Galileo, as it is currently foreseen for future GBAS architectures [4].

A similar analysis could be performed when using GLONASS or BeiDou as a second constellation as this work uses Galileo as an example. Moreover, the possibility of including all the constellations available up to four (GPS, GLONASS, Galileo and BeiDou) as well as the information provided by SBAS could be also considered. However, as shown in [5] the use of more than 2 constellations does not bring any additional benefit as the locations of the satellites in the sky are quasi-redundant and the geometry of the combined constellation does not improve any further.

\section{GBAS PROTECTION LEVEL BACKGROUND}

\section{A. VPL calculation}

One of the main goals of GBAS is to provide integrity assurance. This is achieved by calculating so-called protection levels as position error bounds at the defined integrity risk probabilities (2.0 $\times 10^{-7}$ per approach) [6]. They bound nominal conditions (H0), single-reference-fault conditions (H1) and ephemeris fault conditions (H2). For these conditions, airborne GBAS receivers compute lateral and vertical protection levels (LPL/VPL) in real-time and compare them to the respective alert limits (LAL/VAL) to decide whether or not the operation is safe. In case the protection levels exceed the alert limits, GBAS is set to unavailable.

As the VPL is usually larger than the LPL while the VAL is smaller than the LAL, our assessments are based on the VPL as the measure to compute GBAS availability. According to [6], we set the VAL to 10 meters. The vertical protection level in fault-free conditions $\left(V P L_{H 0}\right)$ is calculated at each epoch using Equation 1 [7]. 1

$$
V P L_{H 0}=k_{f f m d} \cdot \sqrt{\sum_{i=1}^{N} s_{v e r t, i}^{2} \cdot \sigma_{i}^{2}}+D_{V}
$$

Index $i$ indicates the $\mathrm{i}$-th satellite of the $N$ satellites used for the VPL computation. The fault-free missed detection

\footnotetext{
${ }^{1}$ The protection levels in single-reference-fault conditions and ephemeris fault conditions are not used in this work because they apply to different failure modes, which are not considered in this paper.
}

multiplier $k_{f f m d}$ is set to 5.81 according to [7], fulfilling the above mentioned integrity constraints for a base station with three reference receivers. $D_{V}$ is the vertical difference between a position solution based on 100 seconds smoothed pseudoranges and another position solution based on $30 \mathrm{sec}-$ onds smoothed pseudoranges. It is not considered in this study as it depends on the active approach type and the ionospheric conditions during the approach. $\sigma_{i}^{2}$ is the variance of a normal distribution that overbounds the true postcorrection rangedomain error distribution for satellite $i$ under the fault-free hypothesis. The components of $\sigma_{i}^{2}$ are:

$$
\sigma_{i}^{2}=\sigma_{p r \_g n d, i}^{2}+\sigma_{\text {tropo }, i}^{2}+\sigma_{p r_{\_} a i r, i}^{2}+\sigma_{\text {iono }, i}^{2}
$$

where $\sigma_{p r_{-} g n d, i}$ is the total fault-free standard deviation of the error included in the differential corrections for satellite $i$, $\sigma_{\text {tropo }, i}$ is the standard deviation of the error term associated with residual tropospheric uncertainty for satellite $i, \sigma_{p r \_a i r, i}$ the corresponding error term that bounds fault-free airborne receiver multipath and noise errors for satellite $i$, and $\sigma_{i o n o, i}$ accounts for the nominal residual ionospheric uncertainty for satellite $i . s_{\text {vert }, i}$ is calculated as follows [7]:

$$
s_{\text {vert }, i}=s_{3, i}+\tan \left(\theta_{G P A}\right) \cdot s_{1, i}
$$

with the glide path angle $\theta_{G P A}$ equal to $3^{\circ}$ and $s_{1, i}$ and $s_{3, i}$ corresponding to the first and the third rows of the pseudoinverse $S$ of the weighted geometry matrix $G$ containing all the available satellites which can be written as:

$$
S=\left(G^{T} W G\right)^{-1} G^{T} W
$$

Each row $G_{i}$ of $\mathrm{G}$ is defined as:

$$
\left[-\cos \left(E l_{i}\right) \cos \left(A z_{i}\right)-\cos \left(E l_{i}\right) \sin \left(A z_{i}\right)-\sin \left(E l_{i}\right) \quad 1\right]
$$

with $E l_{i}$ and $A z_{i}$ being the elevation and azimuth of the i-th satellite respectively.

The inverse of the weighting matrix is defined as:

$$
W^{-1}=\left(\begin{array}{ccc}
\sigma_{1}^{2} & \cdots & 0 \\
\vdots & \ddots & \vdots \\
0 & \cdots & \sigma_{N}^{2}
\end{array}\right)
$$

where $\sigma_{1}^{2} \ldots \sigma_{N}^{2}$ are the ones calculated in Equation 2.

\section{B. General simulation settings}

All the performed VPL computations in this paper are based on the simulation settings listed in the following unless otherwise stated:

- Orbit parameters:

- GPS: the broadcast almanacs of 27 usable GPS satellites.

- Galileo: the nominal future orbits of 27 Galileo satellites.

- Baseline geometry: the GPS constellation of 27 satellites with a $5^{\circ}$ elevation mask. 


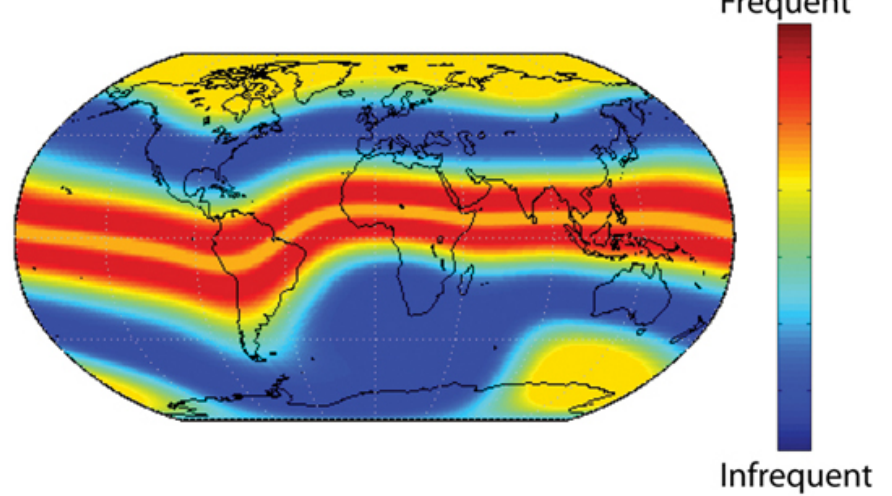

Fig. 1: Scintillation map showing the frequency of disturbances at solar maximum [10]. The intense dark red color shows the locations in which scintillations occur more frequently and the dark blue locations in which scintillations are not likely to occur.

- Temporal sampling: one sample per 100 seconds.

- $5 \mathrm{~km}$ distance of a user to the GBAS reference point.

- $15 \mathrm{~m}(\cong 50 \mathrm{ft})$ height difference to the GBAS reference point.

- Aircraft velocity of $70 \mathrm{~m} / \mathrm{s}$.

- $\sigma_{\text {pr_gnd }}$ values calculated from measurements of DLR GBAS testbed in Braunschweig, Germany ([8],[9]).

\section{Simulation OF IONOSPHERIC ANOMALIES}

\section{A. Description of ionospheric anomalies}

\section{Scintillation}

Scintillations occur in the propagation of the GNSS signals through plasma density irregularities in the ionosphere leading to observed power fades of up to $30 \mathrm{~dB}$. Most receivers will not be able to track these signals resulting in loss of lock of the affected satellites. Scintillations are mostly observed in two bands north and south of the geomagnetic equator and are very rarely seen in mid-latitudes [10] as shown in Figure 1.

\section{Ionospheric plasma bubbles}

Scintillation usually occurs around the so-called ionospheric plasma bubbles. The term plasma bubble is used to describe a region in the nighttime ionosphere within which the plasma density is significantly reduced. Characteristics of these bubbles such as temporal evolutions and vertical, latitudinal and longitudinal development have been studied extensively using different techniques. In [11], the authors study the motion of large scale ionospheric plasma bubbles as well as smaller sized plasma irregularities in North-South and East-West directions using an all-sky imaging system situated at a low latitude station, Kolhapur in India. It has been observed that the EastWest extension of these bubbles varies from 50 to $250 \mathrm{~km}$ and the North-South dimension is larger than $1000 \mathrm{~km}$. Another important feature of these bubbles is the velocity at which they move. In this direction, several studies have been carried out. In [11], the authors estimate the value of $140 \mathrm{~m} / \mathrm{s}$ for the eastward component of the velocity of the plasma bubble at Kolhapur, India. In [12], a more detailed study on the velocity of plasma bubbles is performed. They conclude that the velocity may increase from $112 \pm 10 \mathrm{~m} / \mathrm{s}$ in the evening hours to reach a maximum of about $165 \pm 30 \mathrm{~m} / \mathrm{s}$ around the sunset. Then, it decreases with time. In [13], the zonal constant value of $100 \mathrm{~m} / \mathrm{s}$ is assumed for the eastward component of the velocity of the plasma bubble. In this work, we set the velocity to $100 \mathrm{~m} / \mathrm{s}$ for our simulations.

Besides these characteristics, other features have to be taken into account. In [14], ionospheric TEC maps produced by using 130 ground-based GPS receivers and all-sky images produced by airglow imagers show that usually bubbles do not occur alone, but typically in sequences of 4 to 6 bubbles. The horizontal spacing between two bubbles was found to be approximately 600 to $700 \mathrm{~km}$ over the South American continent. In [15], equatorial plasma bubbles and broad depletions are studied by analyzing the measurements collected by C/NOFS satellites. The authors report that the growth duration of the bubble is more than 3.3 hours and after its formation it may stay in the sky for more than 3.3 hours. They measure different East-West sizes of the bubble pointing out that its normal width in longitude is approximately $100 \mathrm{~km}$. The possibility of these bubbles to merge if the space between successive bubbles in a sequence is less than the width of one bubble in longitude, in this case $100 \mathrm{~km}$, is also investigated. The authors found that bubbles could merge forming wide bubbles until $700 \mathrm{~km}$ width in longitude or broad plasma depletions with sizes of $2200 \mathrm{~km}$.

The aforementioned characteristics change from one location to another and that is why they are referred in some of these works as zonal characteristics. In all of the locations where the appearance of plasma bubbles is common, the hours after sunset are the most critical times for them to develop.

\section{B. Simulations}

\section{IPP}

In order to determine the impact of an ionospheric plasma bubble on the performance of current GBAS, it is necessary to calculate which satellites are affected by it. For this purpose, we use the ionospheric thin-shell model, assuming the ionosphere to have all its TEC (Total Electron Content) concentrated in a layer infinitely thin at $350 \mathrm{~km}$ height from the surface of the Earth. This way, we suppose that the bubble is moving throughout this layer. With the purpose of identifying which pseudoranges coming from the satellites to the receiver go through an altered ionosphere with irregularities, we calculate the ionospheric pierce points (IPPs). The ionospheric pierce point (IPP) is defined to be the intersection of the line segment from the receiver to the satellite and an ellipsoid with constant height of $350 \mathrm{~km}$ above the WGS84 ellipsoid. We assume that when these IPPs are affected by the bubble, the pseudoranges coming from these satellites experience strong scintillations and the receivers lose track of them. After the bubble has passed, the visible satellites are 


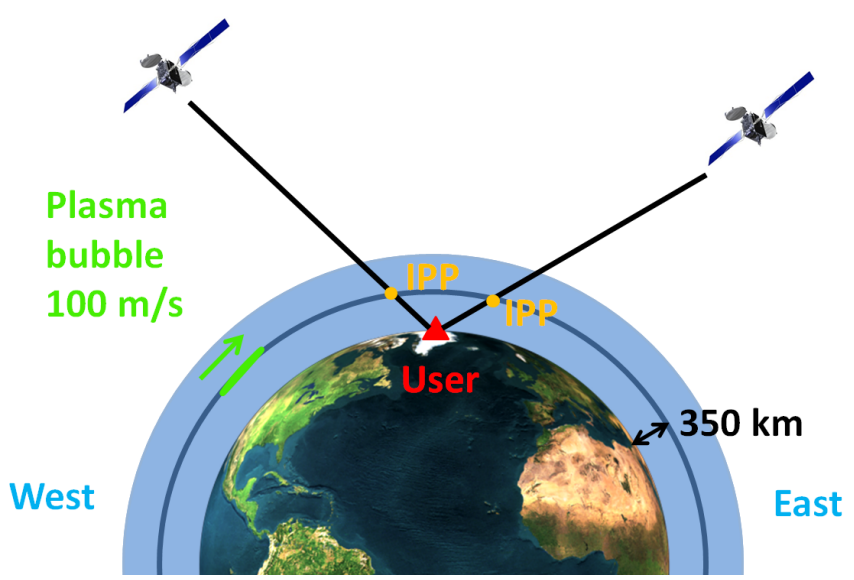

Fig. 2: Diagram of the ionospheric pierce points and the movement of the plasma bubble.

assumed to be tracked and used again. A diagram of the IPP and the movement of the plasma bubble is shown in Figure 2.

\section{Simulation scenarios and results}

In this subsection, we show the results of assuming a second constellation available, Galileo, for navigation during plasma bubble events. We simulate two different scenarios in order to better investigate the impact of ionospheric bubbles on GBAS availability.

\section{Scenario 1: a single moving plasma bubble.}

The first scenario consists of a single bubble moving across the visible area of the thin shell layer, as shown in Figure 2, from the aircraft flying $5 \mathrm{~km}$ away from the GBAS ground station. Only when the bubble is out of the visible part of the sky, another bubble rises over the horizon again such that only one bubble at a time is affecting the user.

We assume the following parameters for the simulation of the bubble:

- Shape: rectangular.

- East-West dimension: variable from 0 to $250 \mathrm{~km}$ in longitude (steps of $50 \mathrm{~km}$ ). We choose these widths in longitude taking into account the results from [11].

- North-South dimension: invariable, $1000 \mathrm{~km}$, centered at the aircraft.

- Bubble speed: eastward $100 \mathrm{~m} / \mathrm{s}$ [13].

Moreover, we consider that the bubble moves eastwards maintaining always the same latitude and changing only its longitude. We perform the simulations in Rio de Janeiro since it is one of the regions that is most affected by the scintillations (22.49 $\mathrm{S}$ latitude and $43.15^{\circ} \mathrm{W}$ longitude) [10].

We illustrate in Figure 3 an example of this first scenario. Here, we show the path of the IPP corresponding to the visible GPS satellites during 7 hours in Rio de Janeiro. We display the PRN of each of the visible GPS satellites in the position of the

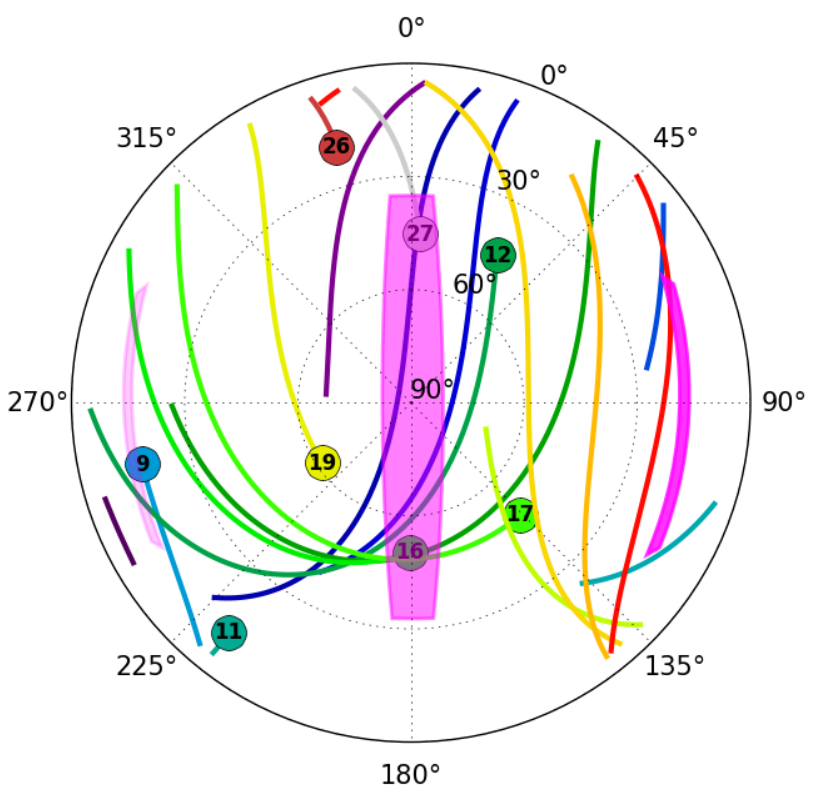

Fig. 3: Representation of the first scenario during only 7 hours of a day in Rio de Janeiro. The lines in different colors show the path followed by the IPP of visible GPS satellites. The PRNs of the corresponding satellites are represented at 4:00 am (GPS Time) and the different shading of the bubble shows its movement in time.

IPP at 4:00 am (GPS Time) as an example. The rectangularshaped bubble is represented in pink, in its projection in polar coordinates. The bubble is shown in three stages: the moment in which it appears in the visible area of the sky, the instant when it is right above the reference point, and the moment just before leaving the visible area of the sky. These stages are represented with a different shading of the bubble, from the brightest (first stage) to the darkest (last stage) in Figure 3.

As we have mentioned previously, these bubbles do not occur during the day, but after sunset. Theoretically, GPS constellation repeats every 11 hours and 58 minutes and each of its satellites pass over the same place on Earth (ground track repeatability) every sidereal day ( 23 hours 56 minutes). The same geometry occurs every day but shifted by 4 minutes with respect to the day before. On the other hand, Galileo satellites have a ground track repeatability of 10 sidereal days and consequently, there is no repeatability each day. For that reason, we do not limit our simulations to just the hours after sunset. In order to consider a great number of possible combinations for the actual satellite geometries in each time stamp we simulate the effect of the bubble during one full day for GPS and during 10 days for the GPS and Galileo constellations combined.

We analyze the impact of the simulated bubble on the availability of GBAS. Here, we compute the VPL every 100 seconds using the visible satellites that are not covered by the bubble. First, we consider GPS constellation alone. Second, 


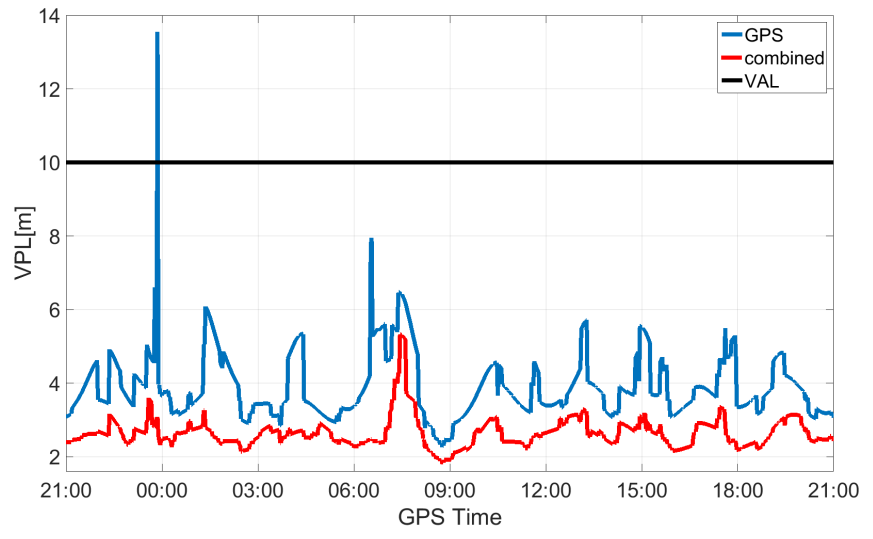

Fig. 4: Impact on the VPL calculation of a $250 \times 1000 \mathrm{~km}$ single plasma bubble. The black line is the VAL, which is set to $10 \mathrm{~m}$. GPS baseline geometry (blue) is compared with GPS and Galileo constellations combined (red).

\begin{tabular}{|c|c|c|}
\hline \multirow{2}{*}{$\begin{array}{c}\text { Bubble longitudinal } \\
\text { dimension }[\mathbf{k m}]\end{array}$} & \multicolumn{2}{|c|}{ VPL [m] } \\
\cline { 2 - 3 } & GPS & GPS+Galileo \\
\hline $\mathbf{0}$ & 3.17 & 2.19 \\
\hline $\mathbf{5 0}$ & 3.84 & 2.43 \\
\hline $\mathbf{1 0 0}$ & 4.96 & 2.47 \\
\hline $\mathbf{1 5 0}$ & 5.16 & 2.78 \\
\hline $\mathbf{2 0 0}$ & 6.61 & 2.78 \\
\hline $\mathbf{2 5 0}$ & 13.55 & 3.08 \\
\hline
\end{tabular}

TABLE I: VPL for different bubble sizes at 23:51 (GPS Time)

we simulate the combined constellation of GPS and Galileo satellites. Then we compare the VPL with the VAL, which is set to 10 meters as mentioned in Section II. If the VPL exceeds the VAL, the GBAS is set to unavailable. As for the combined constellation the ground repeatability is 10 days, we simulate 10 days and choose the worst possible day in terms of VPL (the day with the highest VPL) to compare it with GPS alone. The results of this study considering a plasma bubble of 250 $\mathrm{km}$ width in the longitudinal dimension and $1000 \mathrm{~km}$ length in latitude are shown in Figure 4.

The decrease in the VPL value is clearly visible in the dual constellation case. While a single plasma bubble of $250 \times 1000 \mathrm{~km}$ would cause an unavailability of the single constellation system during a period of 300 seconds, the combined constellation provides availability during the full day. However, although the values presented are calculated for the biggest size of the bubble, other sizes (from $50 \mathrm{~km}$ to $200 \mathrm{~km}$ in steps of $50 \mathrm{~km}$ width in longitude and $1000 \mathrm{~km}$ length in latitude) have been simulated as well. In Table 1, we show the VPL values for different bubble sizes at the time stamp where the VPL maximum occurs (23:51 GPS Time).

We observe that the VPL for the single-constellation case is strongly dependent on the bubble dimension. However, the inclusion of a second constellation reduces the VPL variation

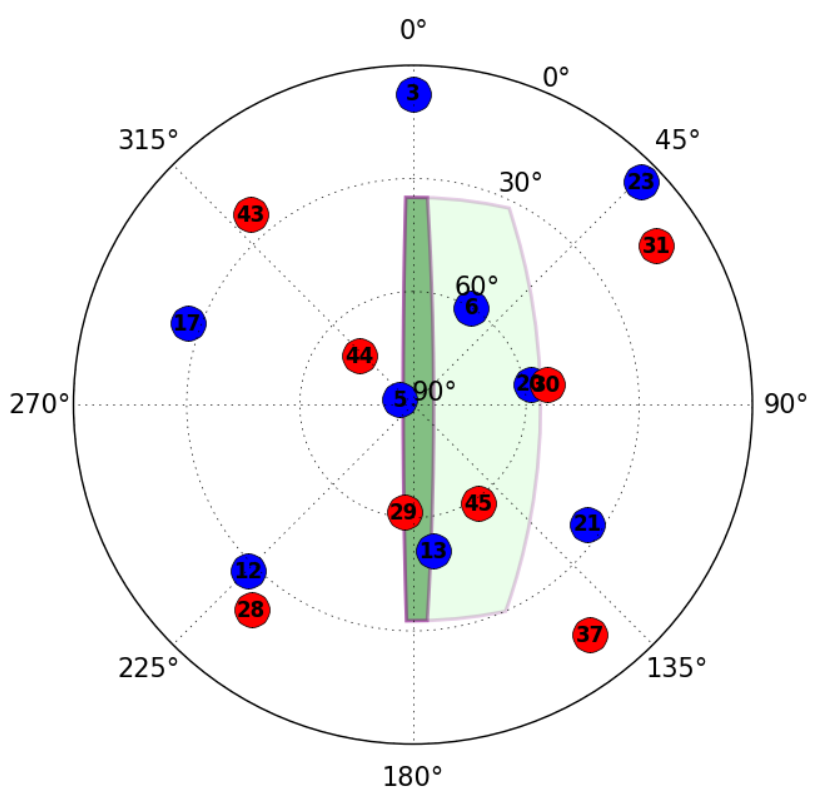

Fig. 5: GPS (blue and indexes from 1 to 27) and Galileo (red and indexes from 28 to 54) satellites represented at the time stamp where the maximum of the VPL occurs (23:51 GPS Time). The $50 \times 1000 \mathrm{~km}$ bubble is represented in dark green whereas the $250 \times 1000 \mathrm{~km}$ bubble is represented in light green.

considerably. This is due to the fact that if a satellite is affected and therefore excluded from the position calculation, it is likely that it has not much impact as we remove from a greater amount of satellites and the satellite geometry left is still favorable. For a better understanding, in Figure 5, we represent both GPS (blue and indexes from 1 to 27) and Galileo satellites (red and indexes from 28 to 54) and two different bubbles (50 $\mathrm{km}$ width bubble in dark green and $250 \mathrm{~km}$ width bubble in light green) at this critical instant of time (23:51 GPS Time). The situation in Figure 5 taking into account only the light green bubble corresponds to the situation at the maximum of Figure 4.

At this particular time, important satellites in terms of their contribution to the position solution are excluded. Index 5 situated approximately at $90^{\circ}$ is affected by a single plasma bubble of $50 \mathrm{~km}$ width in the single constellation case. This corresponds to a VPL increase of 0.67 meters. In the case of using GPS and Galileo satellites, indexes 5 and 29 are affected and the VPL increase is only about 0.25 meters. In both situations, single and dual constellation, we still dispose of enough high elevation satellites and a favorable geometry left to compute the position solution. When affected by a $250 \mathrm{~km}$ wide bubble, indexes 5, 13, 6 and 20 are within the critical area in the single constellation situation and therefore, removed. Excluding all the high elevation satellites implies a VPL increase of 10.38 meters, resulting in unavailability of the service. In the case of dual constellation, indexes 5, 6 , 13, 20, 29 and 45 are affected. The increase in the VPL is only 0.89 meters. This is due to the fact that high elevation 
satellites, indexes 30 and 44, are still available and thus a rather favorable geometry.

We can observe in both cases, single and dual constellation, that the main differences between bubble sizes are connected with the number of high elevation satellites that are affected by it: in general, the wider the more. However, the number of important satellites that are excluded depends on two factors: the location of the bubble in the sky and the geometry present at each time. Next, we study the dependency of the VPL value on these two factors.

First, we analyze the dependency of the VPL on the elevation of the bubble and the loss on high elevation satellites. As we previously mentioned, we generate a satellite geometry every 100 seconds during one full day for GPS and 10 days for the combined constellation of GPS and Galileo. We compute the VPL for each of the considered satellite geometries assuming a $250 \times 1000 \mathrm{~km}$ bubble that is situated in a fixed position in the sky. Once we compute all the VPLs corresponding to all the satellite geometries and one bubble position, we modify the position of the bubble. The position of the bubble is varied in bins of $1^{\circ}$ in elevation each time, from $1^{\circ}$ to $90^{\circ}$. Then, the increase of the VPL (the difference between the VPL with and without the bubble) is calculated for every satellite geometry inside each degree bin. Finally, we compute the average of the increases of VPL for each position of the bubble in elevation. For example, if we want to calculate the average value of the VPL increase for a single constellation case while a plasma bubble of $250 \times 1000 \mathrm{~km}$ is located at $70^{\circ}$ of elevation, we situate this bubble in a way that the middle point of its rectangular shape is placed at $70^{\circ}$ of elevation. Then, we compute the VPL for every geometry considered of GPS satellites only in this case, taking out the satellites inside the limits of the bubble. After that, we compute the VPLs using all the visible GPS satellites for every geometry and subtract them from VPLs with the affected satellites removed. Finally, we average all these VPL increases and the result is represented in blue at $70^{\circ}$ in Figure 6. The results taking into account all the bubble elevations are presented in Figure 6 .

Figure 6 shows a significant increase of the VPL in mean. The maximum increase is 1.85 meters and it is achieved when the bubble is situated at $83^{\circ}$ in elevation. This value is more than double the maximum achieved by the combined constellation curve, 0.79 meters. Therefore, the exclusion of high elevation satellites due to a bubble is less critical for the availability of the system in the dual constellation case. On the other hand, for the single constellation case, we observe a steep increase of the VPL value while situating the bubble in high elevations and consequently excluding some or all of the high elevation satellites. Besides that, we remark that Figure 6 shows an average value of the VPL increase over elevation, which means that we can encounter values below it (better for the availability of the system) or above it like the peak in Figure 4, causing that the system is unavailable.

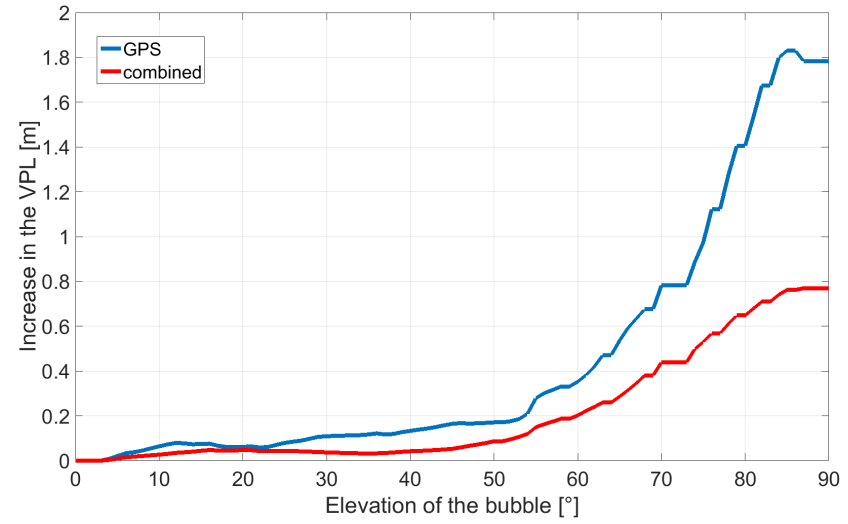

Fig. 6: Mean of the VPL increase depending on the elevation of a single plasma bubble of $250 \times 1000 \mathrm{~km}$. GPS constellation (blue) compared with the combined constellation (red).

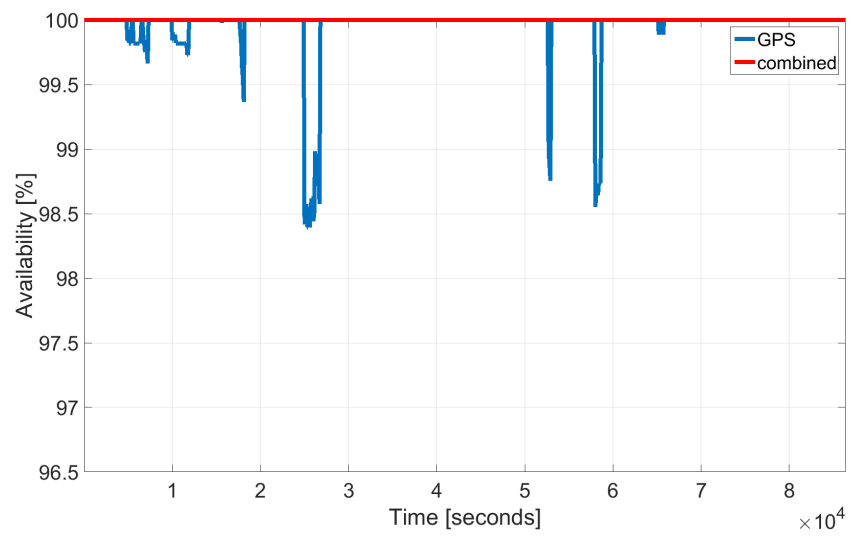

Fig. 7: GBAS availability for a 250 x $1000 \mathrm{~km}$ plasma bubble situated in all the possible locations in azimuth and elevation in steps of $1^{\circ}$. A different geometry is represented by its time of occurrence in the almanac in the $\mathrm{x}$ axis. GPS (blue line) is compared with the combined constellation (red line).

We have analyzed the dependency of the VPL with the bubble position and the exclusion of high elevation satellites. Now, we investigate the dependency of GBAS availability with the geometry of satellites present in the sky while a single plasma bubble of $250 \times 1000 \mathrm{~km}$ is passing through the ionosphere. We simulate satellite geometries for GPS alone and the combination of GPS and Galileo every 100 seconds during 1 and 10 days, respectively. For each instant of time we consider the corresponding geometry and we situate the plasma bubble in all possible locations in elevation and azimuth in steps of $1^{\circ}$. Then, we calculate the percentage of GBAS availability dividing the number of bubble positions which result in a VPL below the alert limit by the total number of the bubble positions considered. As we simulate 10 days for the combined constellation, we choose the worst case in terms of availability (lowest availability) to compare it with GPS alone. The results are presented in Figure 7.

In Figure 7, it can be seen that the minimum value of availability is $98.37 \%$. This means that for this particular GPS 


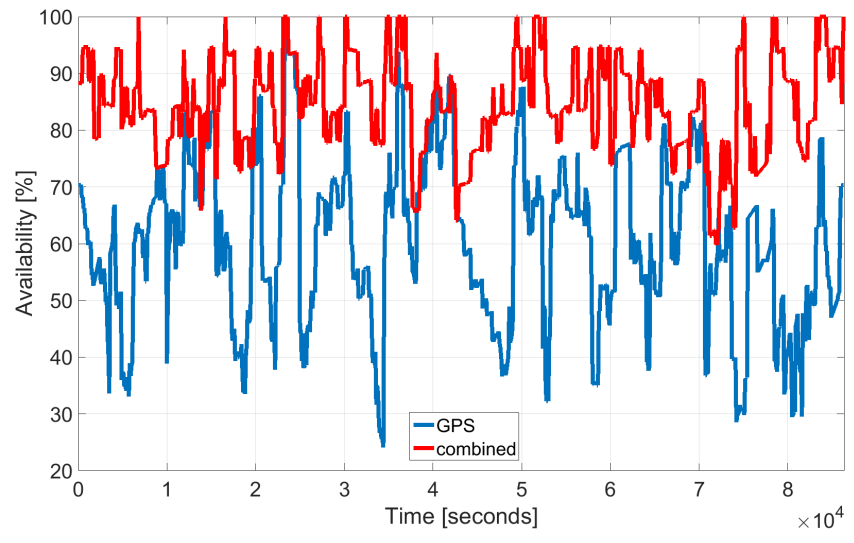

Fig. 8: Average of GBAS availability for a sequence of six plasma bubbles $(250 \times 1000 \mathrm{~km})$ separated $8^{\circ}$ in longitude between them and situated in all the possible locations in azimuth and elevation in steps of $1^{\circ}$. A different geometry is represented by its time in the simulation. GPS (blue line) is compared with the combined constellation (red line).

geometry there are $1.63 \%$ of positions of the bubble in the sky that result in a VPL over VAL. However, the majority of the constellations considered for GPS alone result in a $100 \%$ of availability no matter what the postion of the bubble is. The combined constellation assures GBAS availability $100 \%$ of the times. Therefore, we can conclude that disposing of Galileo as a second constellation improves enormously GBAS availability.

Scenario 2: a moving sequence of plasma bubbles.

In Scenario 1, we have simulated the impact of a single bubble on the GBAS availability. However, these bubbles usually do not come alone, but in sequences of 4 to 6 bubbles separated normally between $600 \mathrm{~km}$ and $700 \mathrm{~km}$ [14]. In Scenario 2, we simulate a sequence of 6 bubbles of $250 \mathrm{x}$ $1000 \mathrm{~km}$ separated $8^{\circ}$ in longitude $(689.425 \mathrm{~km})$. For each satellite geometry considered, we situate a sequence of 6 plasma bubbles in all the possible locations in elevation and azimuth in steps of $1^{\circ}$. The bubbles are always aligned inside the sequence and 6 bubbles are visible. Then, we compute the percentage of GBAS availability dividing the number of sequence positions that results in a VPL below the alert limit by the total number of sequence positions considered. Again, we choose the worst case for the combined constellation in terms of availability (lowest availability) to compare it with GPS alone. In Figure 8, we show the results.

The combined constellation presents higher availability than GPS alone. Galileo as a second constellation provides robustness against unfavorable geometries and consequently, unavailability. GPS alone is overly degraded for every geometry considered and would not be able to guarantee the required GBAS service in the sequence of plasma bubbles case.

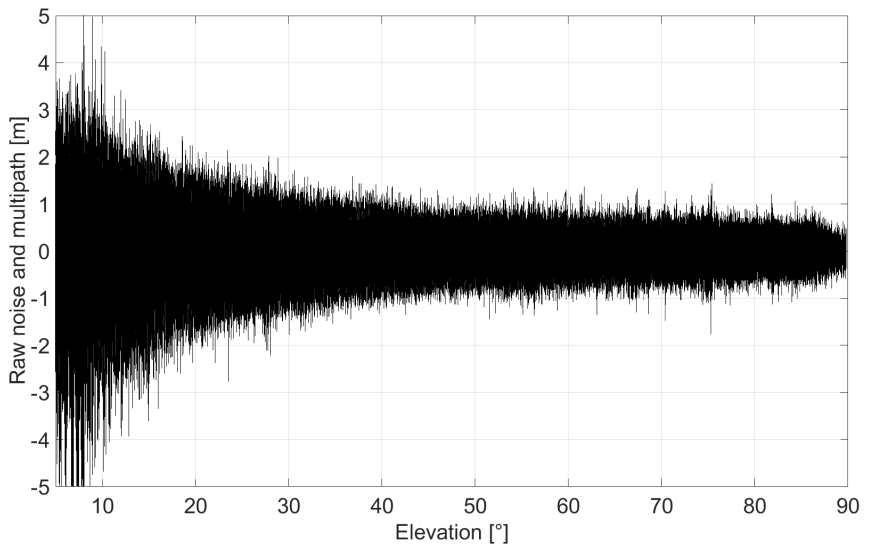

Fig. 9: Raw noise and multipath function of elevation for GPS L1.

\section{Multipath}

A different effect where it might be beneficial to have a second constellation available for navigation is multipath. It represents the dominant nominal error source in satellite-based precision guidance systems. Since multipath errors are not common to the reference station and airborne receivers, they are not eliminated through differential corrections and do not cancel out in a differential GNSS based system like GBAS.

Multipath occurs when GNSS signals are reflected or diffracted from the surrounding terrain, such as ground, buildings, foliage, hills, and the sea surface [16]. In the GBAS context, the aircraft normally operates far enough away of these elements, so the main sources for multipath are the ground reflections and the airframe itself. This means that it mainly affects GNSS signals coming from low elevation angles.

In the particular case of GBAS, the antennas used in the ground segment for certified Category I GBAS stations are multipath limiting antennas. Multipath limiting antennas are optimized to have a very constant gain over all elevations with a sharp cutoff at $5^{\circ}$. In our GBAS testbed in Braunschweig, we use choke ring antennas. Choke ring antennas do not have the same gain pattern but show a clear elevation dependency as we can observe in Figure 9. Figure 9 represents the raw code multipath and noise versus elevation measured by our ground receivers in our GBAS testbed during 10 days. The code multipath and noise was estimated using the linear dualfrequency combination described in [17]. We thus observe in our measurements that our antennas experience high multipath on low elevation satellites.

Besides that, the airborne antenna hardly has any multipath rejection capability so the aircraft experiences multipath for low elevation angles from reflections on the airframe and during roll-out and taxiing also from ground reflections [3]. GBAS parameters already account for multipath. $\sigma_{p r_{-} g n d}$ accounts for multipath coming to the ground station from the 
reflections in the surroundings. $\sigma_{\text {pr_air }}$ accounts for multipath coming to the airborne antenna from the reflections that take place in the aircraft, mainly, in the wings and the tail.

However, GBAS does not have any term that accounts for the multipath in the aircraft produced by ground reflections of the GNSS signals. This kind of multipath affects the aircraft while landing and it is close to the runway and also if we think about future applications such as guided taxiing.

The solution adopted in this work is to raise the elevation mask, which is typically set to $5^{\circ}$. This is a first simple solution and further considerations will take into account more information in order to de-weight the satellites using the $\mathrm{W}$ matrix, giving less importance to that ones in the combined constellation that suffer from high multipath, instead of excluding them. In this study, we raise the elevation mask to $10^{\circ}$ and $15^{\circ}$, which are high enough to eliminate the satellites most affected by multipath. For our simulations, we compute the VPL for every constellation that occurs every 100 seconds in 10 days and compare it with the VAL. After that, we divide the number of cases that are available by the total number of cases and compute the availability for 10 days. We perform these calculations worldwide in a grid of $5^{\circ}$ in latitude by $5^{\circ}$ in longitude.

\section{A. $5^{\circ}$ elevation mask}

The currently used standard masking angle in GBAS is $5^{\circ}$. Under nominal conditions and no further masking, GPS alone has an availability of $100 \%$ in most regions of the world, but it degrades to $99.65 \%$ in two locations in the far North and South. The combined constellation of GPS and Galileo has $100 \%$ of availability.

\section{B. $10^{\circ}$ elevation mask}

Now, we raise the elevation mask to $10^{\circ}$. As we can see in Figure 10, GPS availability degrades down to $96 \%$ in the far North and South regions. Additionally, there are areas in middle latitudes which can also experience some availability problems degrading to $98.21 \%$. The combined constellation has still $100 \%$ availability.

\section{C. $15^{\circ}$ elevation mask}

If the elevation mask is raised to $15^{\circ}$ the availability is degraded further. Figure 11 illustrates that GPS alone has troubles in terms of availability. It degrades to $86.46 \%$ in the northern and southern regions and to $97.85 \%$ in Europe and some parts of the US. The combined constellation still has $100 \%$ availability.

In Table 2, we show the minimum values of the availability of GBAS while raising the elevation mask, additionally to the results presented in Figures 10 and 11. The results show that introducing a second constellation provides sufficient performance and would enable to remove a large portion of the multipath from low elevation satellites by simply raising the elevation masking angle.

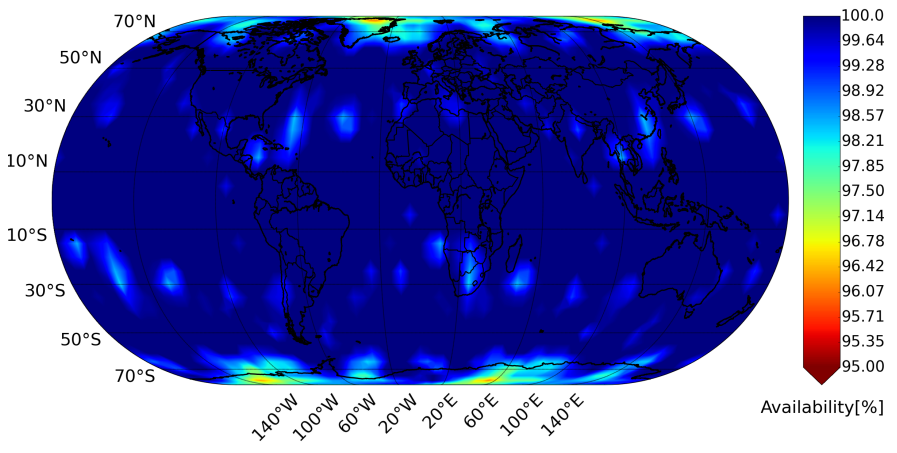

Fig. 10: Availability of GBAS during 10 days using GPS constellation and an elevation mask of $10^{\circ}$. The darkest blue represents the highest availability whereas the dark red represents $95 \%$ of availability or less.

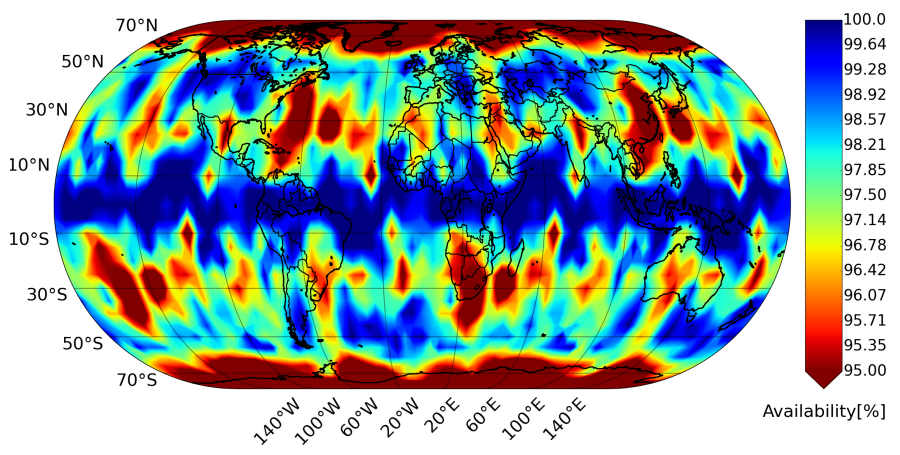

Fig. 11: Availability of GBAS during 10 days using GPS constellation and an elevation mask of $15^{\circ}$. The darkest blue represents the highest availability whereas the dark red represents $95 \%$ of availability or less.

\section{Conclusions}

In this paper we investigated the benefit of using a second constellation in a future GBAS implementation. We considered two potential threats that pose a problem to the availability of the current single-frequency and single-constellation GBAS. Firstly, we analyzed the impact of the plasma bubbles. These bubbles create scintillation effects that cause the receiver to lose lock of the signals passing through the ionosphere. We simulated two different scenarios: (1) a single bubble and (2) a sequence of six bubbles separated $8^{\circ}$ in longitude situated at each possible location in elevation and azimuth in the sky. We assumed that all the satellite signals passing through the bubble were affected and therefore excluded from the position solution. The results show that the combined constellation

\begin{tabular}{|c|c|c|}
\hline $\begin{array}{c}\text { Elevation } \\
\text { mask angle }\end{array}$ & $\begin{array}{c}\text { Min. GPS } \\
\text { availability }\end{array}$ & $\begin{array}{c}\text { Min. combined } \\
\text { availability }\end{array}$ \\
\hline $\mathbf{5}^{\circ}$ & $99.65 \%$ & $100 \%$ \\
\hline $\mathbf{1 0}^{\circ}$ & $96.06 \%$ & $100 \%$ \\
\hline $\mathbf{1 5}^{\circ}$ & $86.46 \%$ & $100 \%$ \\
\hline
\end{tabular}

TABLE II: Minimum availabilities. 
of GPS and Galileo presents higher availability than GPS alone. In the second part of the paper we investigated the possibility to mitigate multipath coming from low elevation satellites by raising the elevation mask to $10^{\circ}$ and $15^{\circ}$. The availability of the GPS-only solution decreases, but the combined constellation provides overall $100 \%$ availability.

As the current single-frequency and single-constellation GBAS will only provide sufficient availability in mid-latitudes, using a second constellation will make the system much more robust against a variety of potential threats.

\section{FUTURE WORK}

In this work we have considered all the satellites in view for both constellations. However, one of the main constraining factors in the definition of a new multi-frequency multiconstellation GBAS concept is the very limited capacity of the VDB link, which is used to provide corrections and integrity parameters to arriving airplanes. Current GPS L1 corrections and integrity parameters still have to be provided in order to allow backwards compatibility (i.e. for aircraft only equipped with legacy GBAS equipment). As a consequence, it is not possible to broadcast all corrections for two frequencies and two (or more) constellations through the current VDB link. Therefore, a selection of a subset of the visible satellites may be needed as proposed in [5].

Additional work will be required in order to analyse the impact of both, scintillations and ground multipath, in dualconstellation GBAS while disposing of not all in view but a subset of satellites.

\section{REFERENCES}

[1] M.-S. Circiu, M. Felux, P. Remi, Y. Lai, B. Belabbas, and S. Pullen, "Evaluation of dual frequency GBAS performance using flight data," PROCEEDINGS OF THE ION ITM, 2014.

[2] T. Fujiwara, "GBAS Availability Assessment and Modelling of Ionospheric Scintillation Effects," Proc. of the ION PNT, 2015.

[3] M. Circiu, M. Felux, B. Belabbas, M. Meurer, J. Lee, M. Kim, and S. Pullen, "Evaluation of GPS L5, Galileo E1 and Galileo E5a Performance in Flight Trials for Multi Frequency Multi Constellation GBAS."

[4] M. Felux and M. Circiu, "Concept for a Dual Frequency Dual Constellation GBAS.” 2015, 16.-18. Sep. 2015, Tampa, FL, USA.

[5] D. Gerbeth, M. Felux, M.-S. Circiu, and M. Caamano, "Optimized Selection of Satellite Subsets for a Multiconstellation GBAS," in Proceedings of the 2016 Interna- tional Technical Meeting of The Institute of Navigation, 2016, pp. 360-367.

[6] N. ICAO, "GBAS CAT II/III development baseline SARPs," International Civil Aviation Organization, 2010.

[7] "RTCA DO-253C. Minimum Operational Performance Standards for GPS Local Area Augmentation System Airborne Equipment. Tech. rep. 253C." Radio Technical Commission for Aeronautics, 2008.

[8] M. Circiu, M. Felux, S. Thölert, F. Antreich, M. Vergara, M. Sgammini, C. Enneking, and S. Pullen, "Evaluation of GPS L5 and Galileo E1 and E5a Performance for Future Multi Frequency and Multi Constellation GBAS," Proc. ION ITM, 2015.

[9] T. Dautermann, M. Felux, and A. Grosch, "Approach service type D evaluation of the DLR GBAS testbed," GPS solutions, vol. 16, no. 3, pp. 375-387, 2012.

[10] P. Kintner, T. Humphreys, and J. Hinks, "GNSS and ionospheric scintillation," Inside GNSS, vol. 4, no. 4, pp. 22-30, 2009.

[11] G. Mukherjee, L. Carlo, S. Mahajan, and P. Patil, "First results of all-sky imaging from India," Earth, planets and space, vol. 50, no. 2, pp. 119-127, 1998.

[12] D. Nade, A. Sharma, S. Nikte, P. Patil, R. Ghodpage, M. Rokade, S. Gurubaran, A. Taori, and Y. Sahai, "Zonal velocity of the equatorial plasma bubbles over Kolhapur, India," 2013.

[13] T. Murphy, M. Harris, and S. Pullen, "Validation of Ionospheric Anomaly Mitigation for GAST D." NAVIGATION SYSTEM PANEL (NSP).

[14] H. Takahashi, C. Wrasse, Y. Otsuka, A. Ivo, V. Gomes, I. Paulino, A. Medeiros, C. Denardini, N. SantAnna, and K. Shiokawa, "Plasma bubble monitoring by TEC map and 630nm airglow image," Journal of Atmospheric and Solar-Terrestrial Physics, vol. 130, pp. 151-158, 2015.

[15] C.-S. Huang, O. de La Beaujardiere, P. Roddy, D. Hunton, R. Pfaff, C. Valladares, and J. Ballenthin, "Evolution of equatorial ionospheric plasma bubbles and formation of broad plasma depletions measured by the C/NOFS satellite during deep solar minimum," Journal of Geophysical Research: Space Physics, vol. 116, no. A3, 2011.

[16] A. Komjathy, V. U. Zavorotny, P. Axelrad, G. H. Born, and J. L. Garrison, "GPS signal scattering from sea surface: Wind speed retrieval using experimental data and theoretical model," Remote Sensing of Environment, vol. 73, no. 2, pp. 162-174, 2000.

[17] M.-S. Circiu, M. Felux, and S. Pullen, "Galileo E1 and E5a Performance for Multi Frequency and Multi Constellation GBAS," GPS World, pp. 30-35, 2015. 